\section{Small intestinal perforation of endoscopically unrecognized lesions during peroral single-balloon enteroscopy}
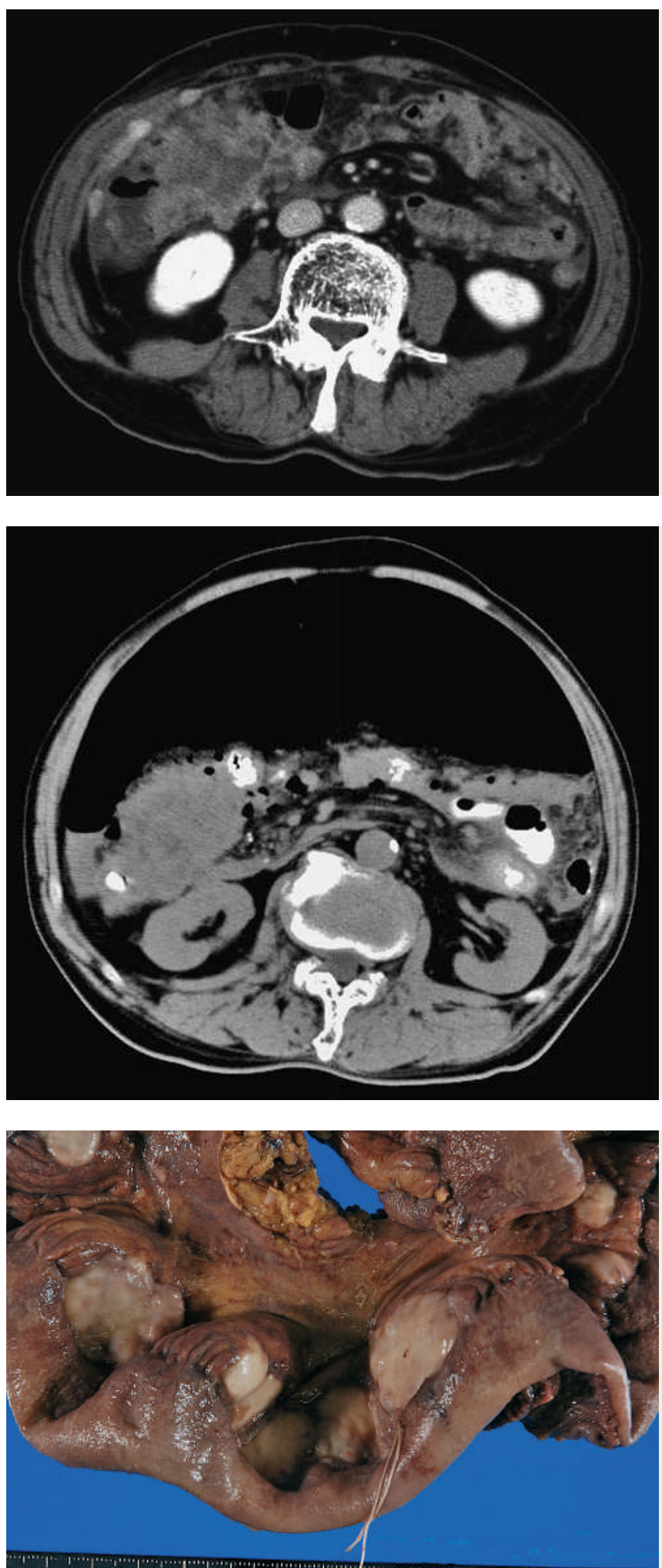

Fig. 1 Computed tomography image showing an abdominal tumor, peritoneal dissemination, and ascites.

Fig. 2 Computed tomography image suggesting perforation of the intestine. Watersoluble contrast medium (meglumine sodium amidotrizoate), injected through the endoscope, can be seen.

Fig. 3 Gross pathological examination showing multiple whitish tumors metastasizing the serosal surface of the small intestine. The site of the perforation is at the top right of a marking suture.
A 79-year-old man presented with a 2month history of abdominal pain. Computed tomography (CT) showed an abdominal tumor, peritoneal dissemination, and ascites ( Fig. 1). Total colonoscopy, including observation of the terminal ileum and esophagogastroduodenoscopy, showed no specific findings. However, because of abnormal radiographic findings of the jejunum, a single-balloon enteroscopy was performed via the anterograde approach using an enteroscope (SIFQ260; Olympus, Tokyo, Japan) with a flexible overtube (ST-SB1; Olympus) [1,2]. The patient was sedated with midazolam (10 mg IV) but complained of abdominal pain during insertion of the endoscope into the jejunum. Perforation was suspected under fluoroscopy before the endoscope had encountered any lesions. The enteroscope was withdrawn and abdominal radiograph and CT scan suggested intestinal perforation ( Fig. 2 ). The small intestine was partially resected because the site of the perforation had been confirmed during the laparotomy. Multiple whitish tumors had metastasized mainly to the serosa of the intestine and peritoneum. Gross pathological examination revealed that metastatic tumors had invaded from the serosa into the mucosa and created multiple ulcers, one of which was perforated ( Fig.3). Histology of the site of perforation revealed a diffuse infiltrate of tumor cells involving all layers of the small intestinal wall ( $\bullet$ Fig. 4). Although the tumors were diagnosed as metastatic carcinoma by further immunohistochemical examination, a diagnosis of metastatic carcinoma of unknown origin was made because further clinical investigations could not confirm the primary site of the carcinoma.

Forceful distention and traction may predispose to perforation of an already weakened small intestinal wall; therefore, if fragile lesions are encountered, insertion of the endoscope beyond the lesions is not recommended $[3,4]$. However, in our case, perforation occurred before the endoscope had encountered the lesions. Although it may be difficult to prevent such problems, care must be taken when using an endoscope in the small 


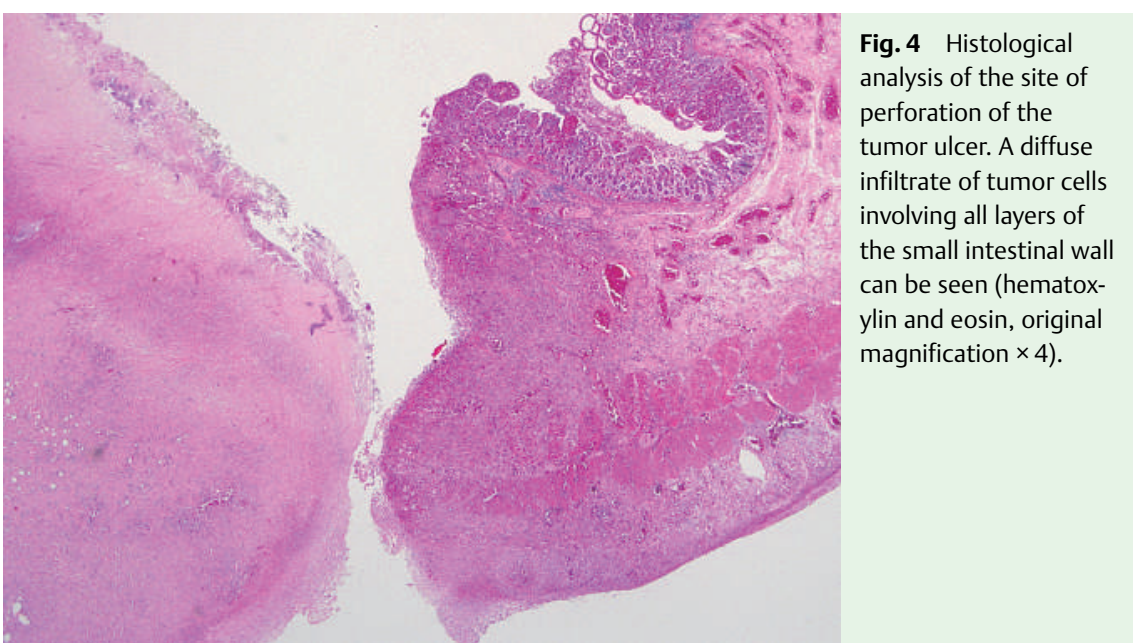

intestine.

Endoscopy_UCTN_Code_CPL_1AI_2AD

K. Tominaga ${ }^{1}$, T. lida ${ }^{1}$, Y. Nakamura ${ }^{2}$, J. Nagao $^{2}$, Y. Yokouchi ${ }^{3}$, I. Maetani ${ }^{1}$

1 Division of Gastroenterology,

Department of Internal Medicine,

Toho University Ohashi Medical Center,

Tokyo, Japan

2 Third Department of Surgery, Toho University Ohashi Medical Center, Tokyo, Japan tinal diseases. Clin Gastroenterol Hepatol 2004; 2: 1010-1016

4 Lo SK. Technical matters in double balloon enteroscopy. Gastrointest Endosc 2007; 66: S15-S18

Bibliography

DOI $10.1055 / \mathrm{s}-2008-1077407$

Endoscopy 2008; 40: E213-E214

(c) Georg Thieme Verlag KG Stuttgart · New York . ISSN 0013-726X

Corresponding author

\section{K. Tominaga, MD}

Division of Gastroenterology

Department of Internal Medicine

Toho University Ohashi Medical Center

2-17-6 Ohashi

Meguro-ku

Tokyo 153-8515

Japan

Fax: 81-3-3468-1269

ktominaga@oha.toho-u.ac.jp 